Human and Animal Health

Vol.60: e17160621, January-December 2017 http://dx.doi.org/10.1590/1678-4324-2017160621 ISSN 1678-4324 Online Edition

\title{
Expression, Purification and Functional Assessment of Smallest Isoform of Human Interleukin-24 in Escherichia coli
}

\author{
Samira Valiyari', Reza Mahdian², Mona Salami ${ }^{3}$, Mana Oloomi ${ }^{1}$, Maryam Golshani ${ }^{1}$, \\ Mohammad Ali Shokrgozar ${ }^{4}$, Saeid Bouzari ${ }^{1 *}$ \\ ${ }^{I}$ Departmant of Molecular Biology, Pasteur Institute of Iran, Tehran, Iran. ${ }^{2}$ Departmant of Molecular Medicine, \\ Pasteur Institute of Iran, Tehran, Iran. ${ }^{3}$ Departmant of Physiology and Pharmacology, Pasteur Institute of Iran, \\ Tehran, Iran. ${ }^{4}$ National Cell Bank of Iran (NCBI), Pasteur Institute of Iran, Tehran, Iran.
}

\begin{abstract}
Interleukin-24 (IL-24) is a novel tumor-suppressor gene that has different alternative splice isoforms. It has been shown that new smallest isoform of human IL-24 gene, lacking three exons, induces higher levels of cytotoxicity than all the isoforms, indicating shortest isoform of IL-24 may be a new promising anti-cancer agent. In this study, we aimed to provide a reproducible method for recombinant production of the smallest isoform of IL-24 (sIL-24). The Structure of sIL-24 was analyzed using bioinformatics tools (I-TASSER, Prosa, RAMPAGE and SPDBV version 4.1). The DNA sequence encoding sIL-24 was chemically synthesized and sub-cloned into the pET-32a (+) vector for further protein expression in Escherichia coli BL21 (DE3) strain. Upon IPTG induction, sIL-24 peptide was expressed as a thioredoxin fusion protein. The recombinant sIL-24 was released from the fusion by TEV protease cleavage followed by nickel affinity chromatography. The yield of the purified sIL-24 was estimated about 380 $\mu \mathrm{g} / \mathrm{ml}$. MTT assay showed that sIL-24 peptide inhibited the proliferation of PC-3 cancer cells more effectively than full length IL-24 protein, while none affect the survival of MRC-5 normal cells. These results indicate that the presented expression system is an efficient system for the production of small functional recombinant sIL-24 peptide.This functional peptide may have cancer therapeutic application.
\end{abstract}

Key words: Smallest isoform of IL-24, Thioredoxin, Recombinant production, Anti-cancer agent

\footnotetext{
*Author for correspondence: saeidbouzari@yahoo.com
} 
Valiyari, $\mathrm{S}$ et al.

\section{INTRODUCTION}

Melanoma differentiation-associated gene 7 (mda-7) is a novel tumor suppressor gene initially identified by subtraction hybridization HO-I human melanoma cell line induced to growth arrest and terminal differentiation ${ }^{1}$. Based on chromosomal location, sequence homology, structure and cytokine-like properties, mda-7 has been named interleukin 24 (IL-24) and classified as a member of the IL-10 cytokine family ${ }^{2-5}$. IL-24 displays at least two separate functions. It mostly acts as a cytokine at normal physiological levels. However, at supra-physiological levels, IL-24 shows an irreversible cancer cell growth suppression and induction of apoptosis, inhibiting invasion, metastasis and angiogenesis, without negative effects on normal cells ${ }^{6-7}$. When IL-24 as a cytokine binds to cell-surface receptors (IL-20R1/IL-20R2 or IL22R1/IL-20R2 heterodimers), Janus activating kinase (JAK) is activated and causes the receptor phosphorylation and creates binding sites for signal transducers and activators of transcription (STAT). However, IL-24 does not depend on JAK/STAT pathway to induce apoptosis ${ }^{8-10}$. Recombinant IL-24 induces genes of the extrinsic and intrinsic apoptotic pathway such as caspase cascade Bax, Bak, Bid, and cytochrome c oxidase ${ }^{11-13}$. Furthermore, IL-24 stimulates the secretion of interferonbeta (IFN-beta), which causes interferon regulatory factor (IRF-1) regulation and Fas/tumour necrosis factor-related apoptosis-inducing ligand (Fas/TRAIL) activation ${ }^{14}$. Numerous studies have demonstrated that IL-24 induces sustained ER stress as evidenced by expression of ER stress markers, such as BiP/GRP78, GRP94, GADD153, XBP-1 and eIF2 $\alpha^{15-18}$. Another mechanism by which IL-24 specifically promotes apoptosis is via oxidative stress. This occurs when IL-24, as transfected into prostate cancer cells, increases the reactive oxygen species (ROS) production followed by mitochondrial dysfunction ${ }^{14,19,20}$. Thus, IL-24 exerts tumor-suppressive effects via multiple signaling pathways. Interleukin-24 gene is comprised of 7 exons and 6 introns with different splice variants reported in the literature ${ }^{21-24}$. A recent study identified and characterized five alternatively mRNA spliced isoforms of tumor suppressor gene IL-24 when expressed in the osteosarcoma cell line U2OS. Several, but not all of these isoforms induce apoptosis in the U2OS cell line, while having no apparent effect on the non-cancerous NOK cell line. Interestingly, one of the new shortest isoforms, mda-7/IL-2482,3,5, which lacks exons 2, 3 and 5, and shares similarity to the C-end of the full-length isoform, represented a consistent ability to induce apoptosis even more effectively than all the isoforms tested, including the full-length mda-7/IL-24 variant. Furthermore, analysis of U2OS cell survival after transient transfection with the original mda-7/IL-24 $2,3,5$ construct and its mutant variants showed that production of protein product of mda-7/IL$24 \delta 2,3,5$ is necessary for its cytotoxic activity ${ }^{25}$. These findings suggest that shortest isoform of IL-24 may be new promising agent for cancer treatment. The mda-7/IL$24 \delta 2,3,5$ isoform is capable of producing a peptide with 48 amino acids and molecular weight of $5.7 \mathrm{kDa}$. There are two broad methods for the production of therapeutic proteins and peptides: chemical synthesis and recombinant production by various heterologous expression systems such as prokaryotic cells, yeast cells, insect cells or mammalian cells. Prokaryotic expression system is more attractive for the production of long peptides (> 35 amino acids) and proteins ${ }^{26}$. The major advantages of choosing prokaryotic expression system are because of its ease of use and cost effectiveness. E. coli is one of the prokaryotic organisms of choice for the production of recombinant proteins and peptides due to its ability to grow rapidly and at high cell density on inexpensive media, its very well-known genetics and easy manipulation ${ }^{27}$. E. coli BL21 (DE3) strain is one of the most used strains for protein expression in both industry and academia. This strain contains the T7 RNA polymerase gene under the control of the lacUV5 promoter. The T7 RNA 
polymerase is expressed by addition of isopropyl-1-thio- $\beta$-D-galactopyranoside (IPTG) to the growth media which induces high levels of target protein expression from T7 promoter driven expression vectors (pET series of plasmids) ${ }^{28}$. Previous studies have shown that full-length IL-24 has the ability to induce significant cytotoxic activity in a wide range of cancer cells without affecting normal cells, including firoblasts, epithelial cells, melanocytes and astrocytes ${ }^{7,29}$. In the present work, to study biological function of recombinant smallest isoform of IL-24 and comparison of its effects to full-length IL-24, we used IL-24 cognate receptors positive prostate cancer cell line PC-3 and normal fibroblast cell line MRC-5. To date there has been no report of the recombinant production of smallest isoform of IL-24.This study is the first report of the recombinant expression and purification of the smallest isoform of human IL-24 in Escherichia coli that provides promising scope for its application as a therapeutic agent.

\section{MATERIALS AND METHODS}

\section{Strains, plasmids and enzymes}

E. coli BL21 (DE3), E. coli TOP10 and plasmid pET-32a (+) were obtained from Novagen, USA. Restriction enzymes and T4 DNA ligase were purchased from Fermentas, USA. TEV protease was purchased from Sigma, Germany.

\section{Bio-informatics Analysis}

mRNA sequence of human full-length IL-24 gene was obtained from NCBI GenBank (www.ncbi.nlm.nih.gov/genbank/) under accession No. NM_001185156.1. Considering that the sIL-24 ORF is predicted to include sequences from exons 6 and 7, we find out nucleotide and amino acid sequences of sIL-24 ORF. The amino acids sequence was analyzed by Expasy MW/pI (web.expasy.org/compute_pi/) to compute the molecular weight and $\mathrm{pI}$ of the peptide ${ }^{30}$. sIL-24 modeling was performed using Threading ASSEmbly Refinement (I-TASSER) server (http://zhanglab.ccmb.med.umich.edu/I-TASSER ${ }^{31}$. Validation and analysis of modeling results were carried out using protein structure analysis (Prosa) server (https://prosa.services.came.sbg.ac.at/prosa.php), Swiss PDB Viewer (SPDBV) software (version 4.1) and Ramachandran Plot Analysis (RAMPAGE) server (http: //mordred.bioc.cam.ac.uk / rapper /rampage.php) ${ }^{32-34}$. The C-score (a confidence score for estimating the quality of predicted model), TM-score (scale for measuring the structural similarity between two structures) and RMSD (known as standards for measuring structural similarity between two structures which are usually used to measure the accuracy of structure modeling when the native structure is known) were given in the I-TASSER output page. Z-score (overall model quality) and energy plots were calculated using Prosa server.

\section{Construction of recombinant expression plasmid}

The coding sequence of sIL-24, sequence of TEV protease (TEVp) cleavage site, BamHI and XhoI endonuclease sites, were chemically synthesized according to codon preference of E. coli. Coding sequence for TEVp site was incorporated at the $5^{\prime}$ end of sIL-24.This sequence was ligated into the expression vector pET-32a at BamHI/XhoI restriction site downstream to the gene of fusion partner thioredoxin (Trx). The resultant recombinant plasmid was named pET32a/sIL-24.The ligation mixture was transformed into E. coli TOP10 a competent cells for propagation of the recombinant plasmid. Transformed bacteria were cultured on LB agar containing 
$100 \mu \mathrm{g} / \mathrm{ml}$ of ampicillin and selected colonies were analyzed for the presence of the recombinant plasmid by PCR, restriction enzyme digestion and DNA sequencing.

\section{Expression of Fusion Protein}

The confirmed pET32a/sIL-24 recombinant plasmid containing fusion gene was transformed into competent $E$. coli BL21 (DE3) cells, and the transformed cells were grown overnight in $5 \mathrm{ml} \mathrm{LB}$ broth (with $100 \mu \mathrm{g} / \mathrm{ml}$ ampicillin) at $37^{\circ} \mathrm{C}$. This overnight culture was used to inoculate $50 \mathrm{ml}$ of fresh LB medium and cells were grown at $37^{\circ} \mathrm{C}$ in a shaker incubator. When the culture OD600 reached 0.5 , the expression of fusion proteins was induced by adding isopropyl-b-Dthiogalactopyranoside (IPTG) (Invitrogen, USA) to a final concentration of $0.2 \mathrm{mM}$. After additional $4 \mathrm{~h}$ cultivation at $160 \mathrm{rpm}$ and $37^{\circ} \mathrm{C}$, the cells were collected by centrifugation at $5000 \mathrm{rpm}$ for $10 \mathrm{~min}$ at $4^{\circ} \mathrm{C}$. The presence of recombinant fusion protein in the induced culture were analyzed by 15\% SDS-PAGE followed by Coomassie brilliant blue staining. In order to test solubility of recombinant fusion protein, the bacterial pellet was obtained from $50 \mathrm{~mL}$ of cell culture following induction with IPTG and resuspended in $5 \mathrm{ml}$ ice-cold phosphate-buffred saline (PBS).After having freeze-thawing in liquid nitrogen and cold water $\left(4^{\circ} \mathrm{C}\right)$, the cells were broken by sonication on ice centrifuged at $12000 \mathrm{rpm}$ for 15 minutes at $4^{\circ} \mathrm{C}$. The presence of fusion protein in the pellet (insoluble fraction) and clear supernatant (soluble fraction) was analyzed on 15\% SDS-PAGE.

\section{Purification of Fusion Protein}

Bacteria from $50 \mathrm{~mL}$ induced culture were pelleted by centrifugation at $5000 \mathrm{rpm}$ for 10 minutes at $4^{\circ} \mathrm{C}$. The bacterial pellet was resuspended in $50 \mathrm{~mL}$ PBS, then lysed by three cycles of freeze-thawing in liquid nitrogen and cold water $\left(4^{\circ} \mathrm{C}\right)$, sonicated for 20 cycles of $20 \mathrm{sec}$, and centrifuged at $12000 \mathrm{rpm}$ for 15 minutes at $4^{\circ} \mathrm{C}$. The pellet was resuspended in buffer B (100 mM NaH2PO4, $10 \mathrm{mM}$ Tris-HCl, $8 \mathrm{M}$ urea; $\mathrm{pH}$ 8.0) containing cocktail of protease inhibitor (Roche, Germany) and incubated for $1 \mathrm{~h}$ at room temperature with shaking to solubilize the inclusion body proteins. The suspension was centrifuged at $12000 \mathrm{rpm}$ for 15 minutes at $4^{\circ} \mathrm{C}$. The clear supernatant was collected and loaded on $1 \mathrm{ml}$ of Ni-NTA resin (Genscript, USA) pre equilibrated with buffer B. The resin-sample suspension was gently agitated at room temperature for $1 \mathrm{~h}$ to allow protein to bind to resin and then centrifuged at $1000 \mathrm{rpm}$ for 5 minutes. The supernatant was removed and the resin was washed with $2 \mathrm{ml}$ of buffer B and buffer C (100 mM NaH2PO4, $10 \mathrm{mM}$ Tris-HCl, $8 \mathrm{M}$ urea; $\mathrm{pH}$ 6.3), respectively. The fusion protein bound with Ni-NTA resin was eluted for five times by adding $1 \mathrm{ml}$ of buffer E (100 mM NaH2PO4, $10 \mathrm{mM}$ Tris-HCl, $8 \mathrm{M}$ urea; $\mathrm{pH}$ 4.5). Fractions corresponding to the fusion protein, as identified by SDS-PAGE, were pooled and refolded by dialysis against decreasing concentrations of urea in 100 bed volumes of $25 \mathrm{mM}$ Tris- $\mathrm{HCl}$ buffer containing $150 \mathrm{mM} \mathrm{NaCl}, 14 \mathrm{mM} \beta$ mercaptoethanol, $10 \%$ glycerol, $0.2 \mathrm{M}$ Arginine, at $4^{\circ} \mathrm{C}$ for $24 \mathrm{~h}$. After dialysis, Protein concentrations were estimated by using the Bradford assay reagent.

\section{Western blot analysis}

Protein samples were subjected to 15\% SDS-PAGE and electrophoretically transferred onto nitrocellulose membrane (Roche, Germany). The membrane was blocked overnight at $4^{\circ} \mathrm{C}$ with $5 \%$ skim milk in TBST buffer $(0.3 \%$ Tris, $0.8 \% \mathrm{NaCl}$, $0.02 \% \mathrm{KCl}$ and $0.1 \%$ Tween 20).Then, the membrane was incubated with anti-IL24 antibody (corresponding to a sequence within amino acids 142-206 of Human IL-24) (Abcam, USA) at 1:1000 dilutions for $2 \mathrm{~h}$ at room temperature. The membrane was washed with TBST buffer for three times and followed by $2 \mathrm{~h}$ incubation with 1:5000 Horse Radish Peroxidase (HRP) conjugated rabbit secondary antibody 
(Abcam, USA). After three washes, the bound antibodies were visualized using DAB (3, 3'- diaminobenzidine tetrahydrochloride) substrate (Sigma, Germany) for HRP.

\section{Proteolytic cleavage of fusion protein with TEV}

To release sIL-24 from the fusion protein, TEVp was added to fusion protein at mass ratio of 1:100 (w/w) according to the manufacturer's instruction .The mixture was incubated at $4^{\circ} \mathrm{C}$ for $16 \mathrm{~h}$. The extent of cleavage of fusion protein was estimated by $15 \%$ SDS-PAGE. The cleavage reaction was then applied to nickel affinity chromatography again to remove Trx-6xHis tag, His tagged TEVp, and residual undigested fusion proteins. The purified recombinant peptide was analyzed by SDSPAGE and western blot.

\section{Functional study of the recombinant sIL-24}

The human prostate cancer cell line PC-3 and normal human fetal lung fibroblast cell line MRC-5 were purchased from National Cell Bank of Iran (Pasteur Institute, Tehran, Iran). The cells were seeded into 96-well tissue culture plates $\left(1 \times 10^{4}\right.$ cells/well) and incubated for $24 \mathrm{~h}$ at $37^{\circ} \mathrm{C}$ and $5 \% \mathrm{CO}_{2}$. The cells were treated with the sIL-24 peptide, recombinant full-length IL-24 protein (Sigma, Germany) and PBS as a negative control at the designated concentrations. After $24 \mathrm{~h}$ treatment, 10 $\mu 1$ of 3-(4,5-dimetylthiazol-2-yl)-2,5-diphenyltetrazolium bromide (MTT) labeling reagent (Roche, Germany) was added to each well. Cells were incubated at $37^{\circ} \mathrm{C}$ and $5 \% \mathrm{CO}_{2}$ for $4 \mathrm{~h}$. Then, $100 \mu \mathrm{l}$ of the solubilization solution was added to each well and followed by incubation at $37^{\circ} \mathrm{C}$ overnight to dissolve formazan crystals. Finally, Absorbance from the plates was read at $570 \mathrm{~nm}$ wavelengths by an ELISA plate reader (Bio Teck, USA). The concentration of sIL-24 peptide that inhibited cell proliferation to $50 \%$ of the control PBS $\left(\mathrm{IC}_{50}\right)$ was calculated from at least three independent experiments in quadruplicate format for each treatment. Percent growth inhibition of treated cells was calculated as follows: \% inhibition=100-(test OD/nontreated OD) $\times 100$ ). Concentration that inhibits $50 \%$ of cancer cell proliferation was used as a parameter for cytotoxicity.

\section{Statistical analysis}

The data represented in this study are mean \pm standard error of the mean (SEM) of at least three identical experiments in quadruplicate replicate. Analysis of variance (ANOVA) followed by a two-tailed unpaired $t$-test was used to assess the significant differences between groups and $p$ values $<0.05$ were considered statistically significant. All analyses were conducted using the IBM SPSS Statistics 20 software. The graphs were created in the software program Excel.

\section{RESULTS}

\section{Bio-informatics Analysis}

In silico prediction of 3D structure of sIL-24 was done using I-TASSER server (Fig. 1A). Validation of the 3D models was performed by related bioinformatics tools. The C-score (-0.23), TM-score (0.68), and RMSD (3.79) of the model indicated that the model has a high confidence value with correct topology. The structure $\mathrm{Z}$-score $(-2.07)$ indicated that the model is within the range of native proteins of the similar size (Fig. 1B). With regard to the Ramachandran plot of the sIL-24, 95.7\% of its residues were in most favored areas (Fig. 1C). The plot of the residues scores 
showed that overall the residue energies were largely negative for the model with the exception of some peaks in the N-terminal part (Fig. 1D).
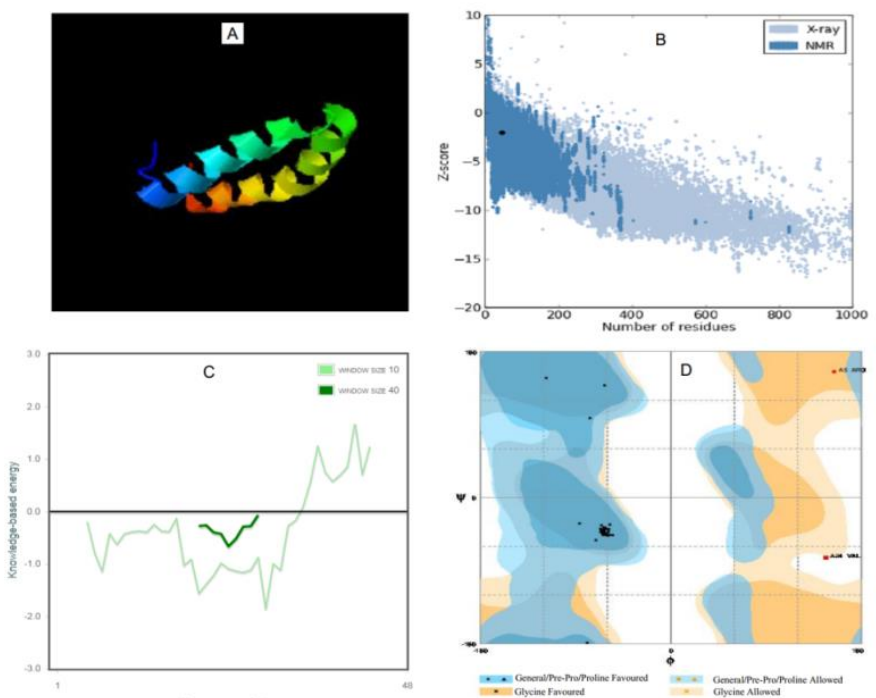

Figure 1 - Results of in silico studies showing (A) sIL-24 protein modeling by I-TASSER server. (B) ProSA-web Z-score plot shows that the sIL-24 protein structure is in the range of scores typically found for native proteins of similar size (the arrow shows the location of the sIL-24). (C) The plot of residue scores. In this plot, overall the residue energies were largely negative for the model. (D) The Ramachandran plot of the sIL-24. Number of residues in favored, allowed, and outer region is $95.7 \%, 0 \%$ and $4.3 \%$, respectively.

\section{Construction of expression vector pET32a/sIL-24}

The sIL-24 coding sequence with an N-terminal TEVp cleavage site and BamHI and XhoI recognition sequences was synthesized. This fragment was then inserted inframe with the $6 \times$ His-tag sequence that is at the carboxyl-terminus of the thioredoxin gene in the fusion expression vector pET-32a The open reading frame of the fusion protein Trx-sIL-24 encodes a protein of 243 amino acids, which consists of a Trx Tag, a 6 His Tag, a S Tag, a 48 residue mature peptide sIL-24 and a TEVp.The pET32a/sIL-24 recombinant vector was confirmed by PCR, restriction endonucleases digestion and DNA sequencing (data not shown). Figure 2 shows a schematic representation of the recombinant fusion protein expression and peptide release strategy.

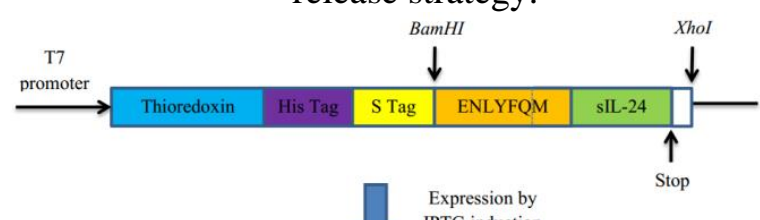

IPTG induction

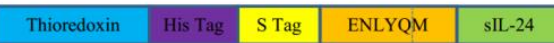

$\int \begin{gathered}\text { Purification by Ni-NTA } \\ \text { resin } \\ \text { TEV protease cleavage }\end{gathered}$
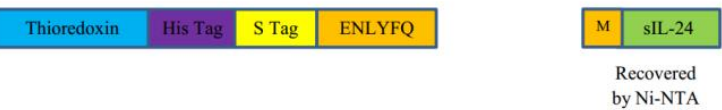

Figure 2 - Schematic representation of expression strategy for the Trx-sIL-24 fusion protein and subsequent TEV cleavage. The arrangement of regions of the construct is shown. sIL-24 peptide released following TEV cleavage has the native sequence as the $\mathrm{N}$-terminal methionine residue is provided by the TEV protease recognition sequence. 


\section{Expression and purification of fusion protein Trx-sIL-24}

E. coli BL21 (DE3) was transformed with pET32a/sIL-24 recombinant expression vector and was induced with IPTG. Theoretically, the molecular weight of the target product of pET32a/sIL-24 should be $26.9 \mathrm{KDa}$. Induced and uninduced cultures were compared by SDS PAGE. A specific band of about $26.9 \mathrm{kDa}$ clearly indicated that the target fusion protein Trx-sIL-24 was expressed (Fig. 3A). To examine the distribution of fusion protein expressed under optimal conditions in soluble and insoluble fractions, both the supernatant and pellet of cell lysate after sonication were analyzed by SDS-PAGE. The majority of Trx-sIL-24 fusion protein was insoluble (Fig. 3B). Therefore, the His-tagged fusion protein was purified under the denaturing condition using nickel affinity chromatography. The identity and purity eluted Trx-sIL-24 was analyzed by SDS-PAGE and Western blot which detected a single band of about $26.9 \mathrm{kDa}$ (Fig. $3 \mathrm{~A}$ and C). The purified Trx-sIL-24 was then thoroughly dialyzed to ensure the removal of all denaturing agents and to allow the protein to acquire a refolded conformation.The expression yield of the purified fusion protein Trx-sIL-24 was determined about $450 \mu \mathrm{g} / \mathrm{mL}$.
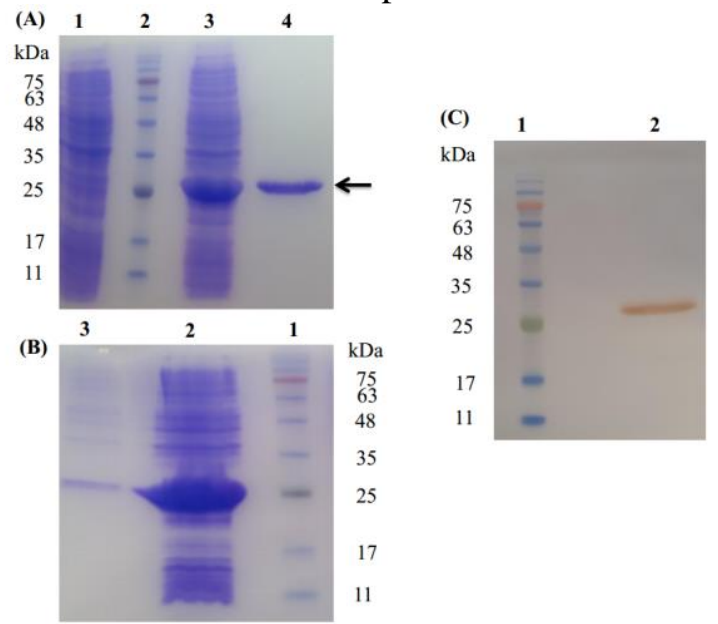

Figure 3 - (A) Expression and purifcation of Trx-sIL-24 fusion protein (indicted by an arrow) as followed by SDS-PAGE (15\%). Lane 1, total protein in un-induced culture; Lane 2, protein molecular weight marker; lane 3, total protein in induced culture; lane 4, the Purified Trx-sIL-24 by Ni-NTA. (B) Solubility testing of fusion protein was analyzed by SDS-PAGE. Lane 1, protein molecular weight marker; Lane 2, inclusion body; Lane 3, soluble total protein. (C) Western blot of the purified fusion protein. Lane 1, protein molecular weight marker; Lane 2, The Purified Trx-sIL-24.

\section{TEV cleavage and preparation of sIL-24 peptide}

To generate recombinant sIL-24 peptide, the purified fusion protein Trx-sIL-24 was treated with TEVp. Reasonably efficient cleavage was achieved after $16 \mathrm{~h}$ incubation at $4^{\circ} \mathrm{C}$ at $100 \mu \mathrm{g}$ fusion protein per unit TEVp. TEV cleavage of the sIL-24 peptide from the Trx fusion protein results in release of sIL-24 with a native N-terminal Methionine provided by the TEV protease site ENLYFQ/M. Two bands, Trx fusion carrier $21.2 \mathrm{kDa}$ and sIL-24 $5.7 \mathrm{kDa}$, are clearly demonstrated in the SDS-PAGE (Fig. 4 A). Following TEV cleavage the sIL-24 peptide was successfully separated from reaction product by nickel affinity chromatography. The yield of the purified recombinant sIL-24 peptide protein was estimated by Bradford assay as $380 \mu \mathrm{g} / \mathrm{mL}$. To confirm the purity of the final sIL-24 product, SDS-PAGE and Western blot was performed and revealed a single band close to $5.7 \mathrm{kDa}$ in excellent agreement with the calculated molecular weight of $5.7 \mathrm{kDa}$ (Fig $4 \mathrm{~A}$ and $\mathrm{B}$ ). 


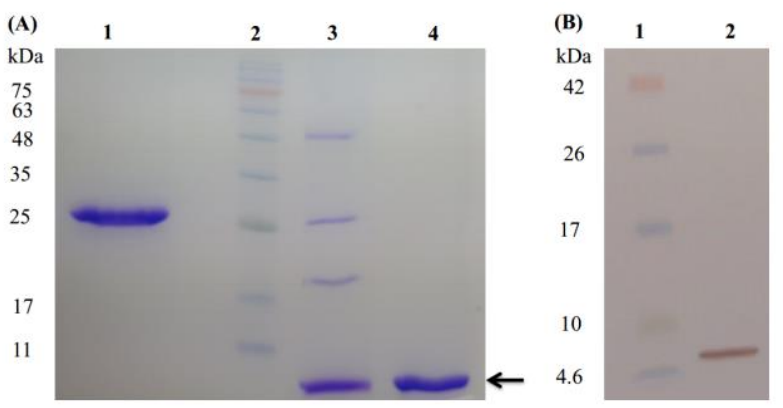

Figure 4 - (A) Peptide release and purification (indicted by an arrow) as followed by SDS-PAGE (15\%). Lane 1, The purified Trx-sIL-24 fusion protein ; lane 2, protein molecular weight marker; Lane 3, Trx-sIL-24 fusion protein after cleavage by TEVp; Lane 4, the purified sIL-24 peptide by Ni-NTA. (B) Western blot of the purified recombinant sIL-24 peptide. Lane 1, protein molecular weight marker. lane 2, the purified sIL-24 peptide by NiNTA.

\section{Functional study of the recombinant sIL-24}

To assess the functional effect of sIL-24, PC-3 and MRC-5 cells were cultured in 96well plates and treated with sIL-24 compared with commercial recombinant fulllength IL-24 at different concentrations. As shown in Figure 5, sIL-24 protein treatment significantly inhibited the PC-3 cell growth. In contrast, no negligible toxicity was observed in control MRC-5 cells. Data analysis of MTT assay showed that $\mathrm{IC}_{50}$ of the sIL-24 and full-length IL-24 in PC-3 cells was $0.48 \mu \mathrm{g} / \mathrm{mL}$ and 1.65 $\mu \mathrm{g} / \mathrm{mL}$ respectively. The data indicate that the sIL-24 could kill cancer cells more powerfully than the full-length IL-24.
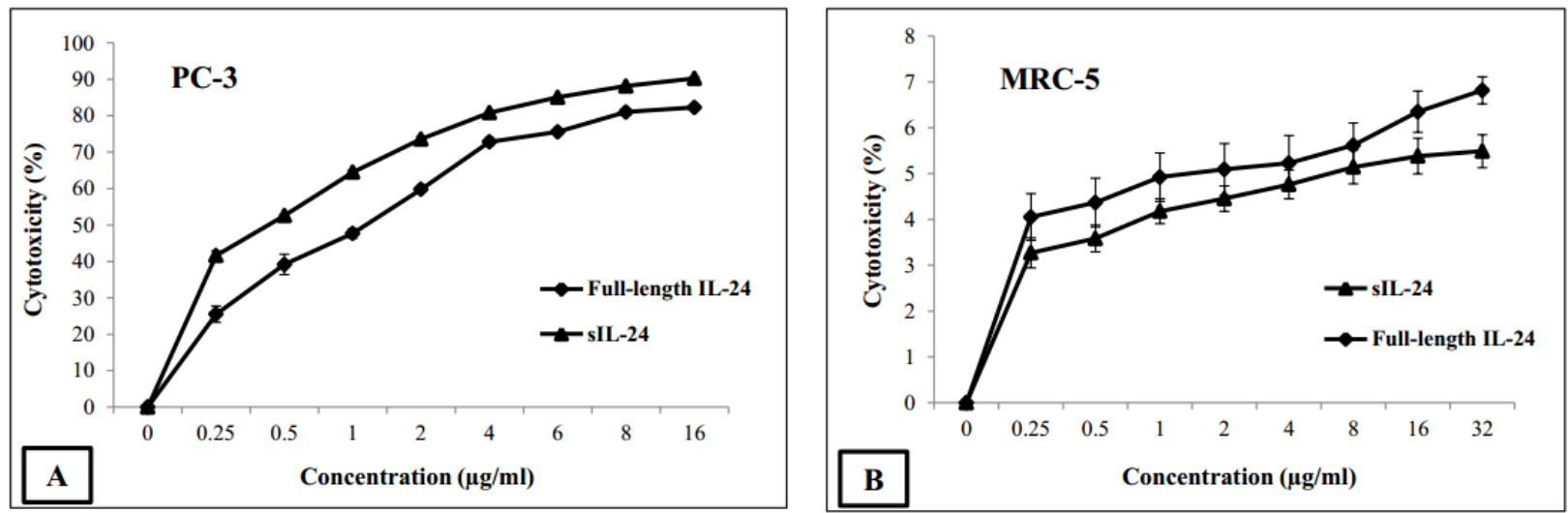

Figure 5 - (A) Cytotoxic effect of sIL-24 on PC-3 cancer cells. PC-3 cells were treated with sIL-24, full-length IL24 and PBS (control) at different concentrations $(0.25,0.5,1,2,4,8,16$ and $32 \mu \mathrm{g} / \mathrm{mL})$ for $24 \mathrm{~h}$. Cell viability was determined by the MTT proliferation assay. (B) Effects of sIL-24 on non-cancerous cell line MRC-5. Data presented are the mean \pm SEM (standard error of the mean) of three independent experiments.

\section{DISCUSSION}

IL-24 has recently received research attention for its ability to selectively induce apoptosis in cancer cells while having minimal effect on normal cells. A recent study characterized and compared the pro-apoptotic properties of five mRNA splice isoforms of the tumor suppressor gene mda-7/IL-24 and found that these mda-7/IL24 isoforms exhibited varying effects on the viability of U2OS cells. Interestingly, a novel shortest isoform, which lacks exons 2, 3 and 5, demonstrated to be the most potent apoptotic inducer of all the isoforms tested, including the full-length mda$7 / \mathrm{IL}-24$ variant. It is known that cytotoxic activity of mda-7/IL-2482,3,5 isoform is due to its small protein product ${ }^{25}$. These results suggest the IL-24 related protein products may lead to new cancer therapeutics. In the present study, we aimed to 
provide a reliable and simple system for recombinant production of the smallest isoform of IL-24 (sIL-24). The open reading frame (ORF) of the sIL-24 generates a peptide with 48 amino acids. Prediction of the 3D structure of sIL-24 using ITASSER, Prosa and Ramachnadran plot indicated that the sIL-24 peptide is with correct topology and within the range of native conformations. The expression of sIL-24 requires fusion partner, since low molecular weight sIL-24 without fusion partner is highly susceptible to proteolytic degradation. A number of fusion partners with different size and properties are available ${ }^{35,36}$. Thioredoxin (Trx) fusion system is widely used for recombinant expression of proteins and peptides in the $E$. coli. Trx's small size $(\sim 12 \mathrm{kDa})$ makes it especially favorable for recombinant peptide production, because it allows the peptide of interest to comprise a relatively large percentage of the fusion ${ }^{37}$. Furthermore, Bogomolovas et al have shown that, among different carrier proteins tested, thioredoxin fusion provided the highest yield of the target peptide ${ }^{38}$. Therefore, to express sIL-24 peptide in E.coli, we used pET-32 a commercial thioredoxin fusion vector. The designed construct containing TEV recognition site and coding sequence of sIL-24 was inserted between the BamHI and XhoI restriction sites of pET-32a. The presence of a His-tag in the fusion facilitates protein purification by affinity chromatography. The analysis of SDS-PAGE and western blotting indicated that Trx-sIL-24 was successfully expressed at high yields in E. coli. However, Trx-sIL-24 fusion protein was unexpectedly expressed in the insoluble form, which is different from many other reports that Trx was a useful fusion partner for expression of soluble proteins. It was also reported that full length of IL-24 24 protein was expressed with Trx fusion partner in the insoluble form ${ }^{39}$. Some properties of the expressed protein, such as post-translational modifications, hydrophobicity, net charge, compositions of compositions of $\mathrm{N}$ - and C-terminal residues and secondary structure are determining factors in the formation of inclusion bodies ${ }^{40}$. Presumably, the expression of full length-IL-24 and sIL-24 in the inclusion body could be related to C-terminal residues. As TrX-sIL-24 fusion protein was insoluble, it is a challenging task to solubilize inclusion bodies and refold the solubilized protein molecules into the native conformation. Since sIL-24 is a small peptide lacking cysteine residues, correct refolding of biologically active of the protein is readily feasible. Moreover, in pET32a $(+)$ system used in this study, the Trx fusion tag expressed from the vector could increase the recovery of soluble target protein isolated from inclusion bodies ${ }^{41}$. After purifcation and renaturation, recombinant fusion protein must be cleaved by enzymes or through chemical reactions to release the peptide in its native form of interest for biological studies. TEV protease is a highly site-specific protease that is found in the Tobacco Etch Virus (TEV). Because its sequence specificity is far more stringent than that of enterokinase, factor Xa or thrombin, TEV protease is a very useful cleavage reagent ${ }^{42}$. In this study, we introduced a TEVp recognition site in the upstream of sIL-24 coding sequense. TEV protease recognizes a 7 amino acid sequence of Glu-AsnLeu-Tyr-Phe-Gln-(Gly/Ser) and cleaves between Gln and Gly or Gln and Ser. A systematic study by Kapust et al. demonstrated that many different amino acids can be incorporated in the P1' position (Gly or Ser) of a TEV protease recognition site with little impact on the processing efficiency ${ }^{43}$. In our study, to obtain sIL-24 without additional N-terminal amino acids, Gly or Ser was substituted with Met. TEV cleavage of the fusion protein was efficiently performed between Gln and Met and resulted in release sIL-24 peptide with a native n-terminal Met. These results support that different P1'variants in the fusion proteins can be substrates for TEVp. As Trx and TEVp include 6xHis-tag and sIL-24 does not contain $6 \times$ His-tag, we could use nickel affinity chromatography to purify target peptide.This allowed the 
more efficient recovery of peptide. Biological activity studies revealed significant cytotoxic activity of sIL-24 in PC-3 cancer cells but not in MRC-5 normal cells. Statistical analysis indicated that cytotoxic efficacy of sIL-24 peptide was more potent than full-length IL-24. Thus, our study confirms previous findings about the shortest isoforms of IL-24. A follow-up investigation of sIL-24 anticancer mechanism is currently underway in our lab.

\section{CONCLUSION}

This study was the first work that considered recombinant production and biological assessment of sIL-24.The results highlight the potential for producing small active peptide such as sIL-24 in a prokaryotic system. The significant cytotoxic activity of sIL-24 peptide in cancer cells suggests that sIL-24 may be a promising source of cancer therapeutic peptides.

\section{ACKNOWLEDGEMENTS}

The authors would like to thank the financial support of Pasteur Institute of Iran.

\section{REFERENCES}

1. Lebedeva IV, Sauane M, Gopalkrishnan RV, Sarkar D, Su ZZ, Gupta P, et al. mda-7/IL24: Exploiting Cancer's Achilles' Heel. Mol Ther. 2005; 11(1).

2. Persaud L, De Jesus D, Brannigan O, Richiez-Paredes M, Huaman J, Alvarado G, et al. Mechanism of Action and Applications of Interleukin 24 in Immunotherapy. Int $\mathrm{J}$ Mol Sci. 2016; 17(6): 869.

3. Imaeda H, Nishida A, Inatomi O, Fujiyama Y, Andoh A. Expression of interleukin-24 and its receptor in human pancreatic myofiroblasts. Int J Mol Med. 2011; 28(6): 993-999.

4. Sauane M, Su ZZ, Gupta P, Lebedeva IV, Dent P, Sarkar D, et al. Autocrine regulation of mda-7/IL-24 mediates cancer-specific apoptosis. Proc Natl Acad Sci. 2008; 105(28): 9763-9768.

5. Sauane M, Gupta P, Lebedeva IV, Su ZZ, Sarkar D, Randolph A, et al. N-Glycosylation of MDA-7/IL-24 Is Dispensable for Tumor Cell-Specific Apoptosis and "Bystander" Antitumor Activity. Cancer Res. 2006; 66(24): 11869-11877.

6. Mirzaei MH, Esmaeilzadeh A. Overexpression of MDA-7/IL-24 as an anticancer cytokine in gene therapy of thyroid carcinoma. J Med Hypotheses Ideas. 2014; 8(1): 7-13.

7. Menezes ME, Bhatia S, Bhoopathi P, Das SK, Emdad L, Dasgupta S, et al. MDA-7/IL24: multifunctional cancer killing cytokine. Adv Exp Med Biol. 2014; 818: 127-153.

8. Andoh A, Shioya M, Nishida A, Bamba S, Tsujikawa T, Kim-Mitsuyama S, et al. Expression of IL-24, an activator of the JAK1/STAT3/SOCS3 cascade, is enhanced in inflammatory bowel disease. J Immunol. 2009; 183(1): 687-695.

9. Su Z, Emdad L, Sauane M, Lebedeva IV, Sarkar D, Gupta P, et al. Unique aspects of mda-7/IL-24 antitumor bystander activity: establishing a role for secretion of MDA-7/IL24 protein by normal cells. Oncogene. 2005; 24(51): 7552-7566.

10. Dent P, Yacoub A, Hamed HA, Park MA, Dash R, Bhutia K, et al. MDA-7/IL-24 as a cancer therapeutic: from bench to bedside. Anticancer Drugs. 2010; 21(8): 725-731.

11. Su ZZ, Lebedeva IV, Sarkar D, Emdad L, Gupta P, Kitada S, et al. Ionizing radiation enhances therapeutic activity of mda-7/IL-24: overcoming radiation-and mda-7/IL-24resistance in prostate cancer cells overexpressing the antiapoptotic proteins bcl-xL or bcl2. Oncogene. 2006; 25(16): 2339-2348.

12. Fisher PB. Is mda-7/IL-24 a "magic bullet" for cancer? Cancer Res. 2005; 65(22): 1012810138. 
13. Tian H, Zhang DF, Zhang BF, Li HZ, Zhang Q, Li LT, et al. Melanoma Differentiation Associated Gene-7/Interleukin-24 Induces Caspase-3 Denitrosylation to Facilitate the Activation of Cancer Cell Apoptosis. J Interferon Cytokine Res. 2015; 35(3): 157-167.

14. Argiris K, Panethymitaki C, Tavassoli M. Naturally occurring, tumor-specific, therapeutic proteins. Exp Biol Med. 2011; 236(5): 524-536.

15. Panneerselvam J, Munshi A, Ramesh R. Molecular targets and signaling pathways regulated by interleukin (IL)-24 in mediating its antitumor activities. J Molec Signal. 2013; 8(1):15.

16. Erfan Manesh M, Esmaeilzadeh A, Hajikhan Mirzaei M. IL-24: A novel gene therapy candidate for immune system upregulation in Hodgkin's lymphoma. J Med Hypotheses Ideas. 2015; 9(1): 61-66.

17. Park MA, Yacoub A, Sarkar D, Emdad L, Rahmani M, Spiegel S, et al. PERK-dependent regulation of MDA-7/IL-24-induced autophagy in primary human glioma cells. Autophagy. 2008; 4(4): 513-515.

18. Park MA, Walker T, Martin AP, Allegood J, Vozhilla N, Emdad L, et al. MDA-7/IL-24induced cell killing in malignant renal carcinoma cells occur by a ceramide/CD95/PERKdependent mechanism. Mol Cancer Ther. 2009; 8(5): 1280-1291.

19. Amirzada MI, Jin J. Therapeutic applications of interleukin 24 (IL24): a review. Trop J Pharm Res. 2013; 11(6): 1023-1027.

20. Li Y, Zhang H, Zhu X, Feng D, Gong J, Han T. Interleukin-24 induces neuroblastoma SH-SY5Y cell differentiation, growth inhibition, and apoptosis by promoting ROS production. J Interf Cytok Res. 2013; 33(11): 709-714.

21. Huang EY, Madireddi MT, Gopalkrishnan RV, Leszczyniecka M, Su ZZ, Lebedeva IV, et al. Genomic structure, chromosomal localization and expression profile of a novel melanoma differentiation associated (mda-7) gene with cancer specific growth suppressing and apoptosis inducing properties. Oncogene. 2001; 20(48): 7051-7063.

22. Allen M, Pratscher B, Roka F, Krepler C, Wacheck V, Schöfer C, et al. Loss of Novel mda-7 Splice Variant (mda-7s) Expression is Associated with Metastatic Melanoma1. J Invest Dermatol. 2004; 123(3): 583-588.

23. Allen M, Pratscher B, Krepler C, Frei K, Schofer C, Pehamberger H, et al. Alternative splicing of IL-24 in melanocytes by deletion of exons 3 and 5. Int J Immunogenet. 2005; 32(6): 375-378.

24. Sahoo A, Jung YM, Kwon HK, Yi HJ, Lee S, Chang S, et al. A novel splicing variant of mouse interleukin (IL)-24 antagonizes IL-24-induced apoptosis. J Biol Chem. 2008; 283(43): 28860-28872.

25. Whitaker EL, Filippov V, Filippova M, Guerrero-Juarez CF, Duerksen-Hughes PJ. Splice variants of mda-7/IL-24 differentially affect survival and induce apoptosis in U2OS cells. Cytokine. 2011; 56(2): 272-281.

26. Kyle S, James KAR, McPherson MJRecombinant production of the therapeutic peptide lunasin. Microb Cell Fact. 2012; 11:28.

27. Joseph BC, Pichaimuthu S, Srimeenakshi S, Murthy M, Selvakumar K, M G, et al. An Overview of the Parameters for Recombinant Protein Expression in Escherichia coli. $J$ Cell Sci Ther. 2015; 6: 221. doi:10.4172/2157-7013.1000221.

28. Rosano GL, Ceccarelli EA. Recombinant protein expression in Escherichia coli: advances and challenges. Front Microbiol. 2014; 5:172. doi: 10.3389/fmicb.2014.0 0172.

29. Gupta P, Su ZZ, Lebedeva IV, Sarkar D, Sauane M, Emdad L, et al. mda-7/IL-24: multifunctional cancer-specifi apoptosis-inducing cytokine. Pharmacol Ther. 2006; 111 : 596-628.

30. Gasteiger E, Hoogland C, Gattiker A, Duvaud S, Wikins MR, Appel RD, Bairoch A. Protein identification and analysis tools on the ExPASy server. In: Walker JM, editor. The proteomics protocols handbook. Totowa: Humana Press Inc; 2005. p. 571-607.

31. Zhang Y. I-TASSER server for protein 3D structure prediction. BMC bioinformatics. 2008; 9(1):40. doi:10.1186/1471-2105-9-40

32. Wiederstein M, Sippl MJ. ProSA-web: interactive web service for the recognition of errors in three-dimensional structures of proteins. Nuc Acids Res. 2007; 35 Suppl 2: W407-W10. 
33. Biasini M, Bienert S, Waterhouse A, Arnold K, Studer G, Schmidt T, et al. SWISSMODEL: modelling protein

tertiary and quaternary y structure using evolutionary information. Nuc Acids Res. 2014; 42(W1): W252-W258.

34. Mannige RV, Kundu J, Whitelam S. The Ramachandran Number: An Order Parameter for Protein Geometry. PLoS ONE. 2016; 11(8): e0160023.

35. Ingham $\mathrm{AB}$, Moore RJ. Recombinant production of antimicrobial peptides in heterologous microbial systems. Biotechnol Appl Biochem. 2007; 47(1):1-9.

36. Costa S, Almeida A, Castro A, Domingues L. Fusion tags for protein solubility, purification, and immunogenicity in Escherichia coli: the novel Fh8 system. Front Microbiol. 2014; 5(63): 1-20.

37. Li Y. Carrier proteins for fusion expression of antimicrobial peptides in Escherichia coli. Biotechnol Appl Biochem. 2009; 54(1): 1-9.

38. Bogomolovas J, Simon B, Sattler M, Stier G. Screening of fusion partners for high yield expression and purification of bioactive viscotoxins. Protein Expr Purif. 2009; 64(1): 1623.

39. Yang J, Zhang W, Liu K Jing S, Guo G, Luo P, et al. Expression, purification, and characterization of recombinant human interleukin 24 in Escherichia coli. Protein Expr Purif. 2007; 53(2): 339-345.

40. Boozarpour S, Sadeghizadeh M, Shokrgozar MA, Hosseinkhani S, Shojaosadati SA, Gharavi S, et al. Bacterial overexpression of the human interleukin-2 in insoluble form via the pET Trx fusion system. Iran J Biotech. 2010; 8(3): 270-274.

41. Da-Hui L, Gui-Liang J, Ying-Tao Z, Tie-Min A. Bacterial expression of a Trichosanthes kirilowii defensin (TDEF1) and its antifungal activity on Fusarium oxysporum. Appl Microbiol Biotechnol. 2007; 74(1): 146-151.

42. Waugh DS. An Overview of Enzymatic Reagents for the Removal of Affinity Tags. Protein Expr Purif. 2011; 80(2): 283-293.

43. Kapust RB, Tözsér J, Copeland TD, Waugh DS. The P1' specificity of tobacco etch virus protease. Biochem Biophys Res Commun. 2002; 294(5): 949-955.

Received: February 03, 2016; Accepted: July 14, 2016 


\section{Erratum}

In Article "Expression, Purification and Functional Assessment of Smallest Isoform of Human Interleukin-24 in Escherichia coli", with DOI number: http://dx.doi.org/10.1590/1678-43242017160621, published in journal Brazilian Archives of Biology and Technology, vol. 60, the 01 page.

That read:

“http://dx.doi.org/10.190/1678-4324-2017160621"

Read:

“http://dx.doi.org/10.1590/1678-4324-2017160621" 